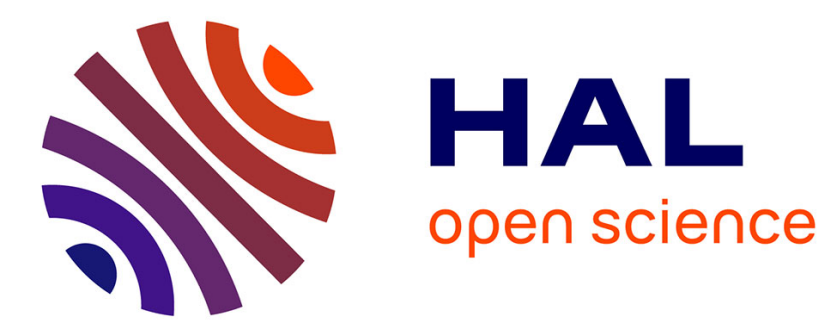

\title{
Fractal Density and Singularity Analysis of Extreme Geo-Processes
}

Qiuming Cheng

\section{To cite this version:}

Qiuming Cheng. Fractal Density and Singularity Analysis of Extreme Geo-Processes. CS-DC'15 World e-conference, Sep 2015, Tempe, United States. hal-01291124

HAL Id: hal-01291124

https://hal.science/hal-01291124

Submitted on 20 Mar 2016

HAL is a multi-disciplinary open access archive for the deposit and dissemination of scientific research documents, whether they are published or not. The documents may come from teaching and research institutions in France or abroad, or from public or private research centers.
L'archive ouverte pluridisciplinaire HAL, est destinée au dépôt et à la diffusion de documents scientifiques de niveau recherche, publiés ou non, émanant des établissements d'enseignement et de recherche français ou étrangers, des laboratoires publics ou privés. 


\title{
Fractal Density and Singularity Analysis of Extreme Geo-Processes
}

\author{
Qiuming Cheng ${ }^{1,2}$, \\ ${ }^{1}$ Department of Earth and Space Science and Engineering, Department of Geography, \\ York University, 4700 Keele Street, Toronto, Ontario, Canada M3K1P3 \\ ${ }^{2}$ State Key Lab of Geological Processes and Mineral Resources, China University of \\ Geosciences, Beijing and Wuhan, China \\ qiuming@yorku.ca
}

\begin{abstract}
Density is a fundamental physical parameter involved in most geodynamics models used for prediction in the earth sciences. A new concept of fractal density was proposed to characterize the extreme physical properties of complex geo-processes. As a generalization of the ordinary density concept, the fractal density has a unit of ratio of mass or energy over a fractal set (e.g. $\mathrm{g} / \mathrm{cm}^{\alpha}$ and $J / \mathrm{m}^{\alpha}$, where the singularity $\alpha$ can be a non-integer number). From a fractal density point of view the ordinary density becomes scale dependent with singularity which should be substituted by fractal density in dynamic models. We demonstrate that several extreme geo-processes occurred in the Earth's crust originated from cascade earth dynamics (mantle convection) and selforganized criticality (slab breakoffs and faults as avalanches) can cause fractal density of mass accumulation or energy release. Examples shown in the paper include energy release of earthquakes and floods.
\end{abstract}

Keywords: power-law, fractal and multifractals, extreme geo-events, mass density and energy density.

\section{Introduction}

Since the principle of density was discovered by the Greek scientist Archimedes approximately 2000 years ago, density has become a fundamental property of mass or energy as a well-known physical concept with a variety of applications. Density as a scale independent property of material or energy has been treated as a fundamental physical parameter and variable of many physical models with applications in nearly all fields of study, ranging from physics to engineering, economics and the social sciences. The density of a material or energy is defined as its mass or energy per unit volume. Therefore, density often is characterized as units of mass over volume (e.g., $\left.\mathrm{g} / \mathrm{cm}^{3}, \mathrm{~kg} / \mathrm{m}^{3}\right)$ or energy over volume $\left(\mathrm{J} / \mathrm{cm}^{3}, \mathrm{w} / \mathrm{L}^{3}\right)$. For example, continental crust, which consists mostly of granitic rock, has a density of about $2.7 \mathrm{~g} / \mathrm{cm}^{3}$ and the Earth's mantle of ultramafic rock has a density of about $3.3 \mathrm{~g} / \mathrm{cm}^{3}$. The density of seawater varies with temperature and salinity of the water. Although the density of seawater varies at different points in the ocean, a good estimate of its density at the ocean's surface is $1025 \mathrm{~kg} / \mathrm{m}^{3}$. Density of air is a temperature and pressure dependent 
parameter. For given temperature and pressure the density of air is independent of the volume of air. Analogy definition of concept of density has been commonly used in many other fields such as in social studies a population density can be defined as a number of people per unit region. Point density as number of points per unit area, and road density as length of roads or a number of road segments per unit area are often seen in spatial analysis. According to the ordinary density the measure or mass within a volume can be estimated as the integral of the density multiplied by the volume. However, from multifracatal point of view the mass or measure defined within a volume can show singularity in which the density measured as the mass over volume may approach to infinite as the volume becomes infinitely small [1]. The ratio of mass over volume does not converge; in this case the density does not exist according to the ordinary definition [2]. In the follow section the new form of density, a generalized form of the above ordinary density will be introduced which quantifies the density of complex objects.

We show that the end products for many types of singular processes can be characterized by fractal mass density or energy density. The concept and index of fractal density will be utilized in dynamic models involved in the modelling of extreme processes [2][3].

\section{Fractal Density and Density-Scale Power-Law Model}

According to the concept of ordinary density, the mass density of an object $(\rho)$ can be calculated by the following equation:

$$
\rho=\frac{m(v)}{V},
$$

where $\mathrm{m}(\mathrm{v})$ represents the mass contained in a volume and $\rho$ is the average density of an object. If the density of the object is homogenous then the density calculated in (1) becomes independent of volume. The unit of the density is determined by the ratio of the mass and volume, for example, $\mathrm{g} / \mathrm{cm}^{3}$. However, if the object has heterogeneous properties, the density may vary from place to place and the average density in (1) varies with different size of $\mathrm{v}$. In this case a localized density must be calculated using the derivative of the mass over volume:

$$
\rho=\frac{d m(V)}{d V}=\lim _{V \rightarrow 0} \frac{m(V)}{V} .
$$

The preceding density exists only if the limit converges when the volume becomes infinitesimal. If the limit does not converge then the density does exist. As a generalization of the equation (2) I introduce a new equation (3) where there exists a parameter $\alpha$ (with positive value) so that the following limit converges:

$$
\rho_{\alpha}=1 \operatorname{im}_{V \rightarrow 0} \frac{m(V)}{V^{\frac{\alpha}{3}}} \text {. }
$$

The value of $\rho_{\alpha}$ can be considered as generalized density because the ordinary density defined in Eq. (2) becomes a special case of Eq. (3) when $\alpha=3$, the normal dimension of volume. This new density is named fractal density which is defined as 
mass or energy per unit "fractal set". The fractal density defined in Eq. (3) has as unit the ratio of mass to a fractal set of $\alpha$ dimensions; for example, $\mathrm{g} / \mathrm{cm}^{\alpha}$ or $\mathrm{kg} / \mathrm{m}^{\alpha}$. Similarly, the units of fractal energy density can be $\mathrm{J} / \mathrm{cm}^{\alpha}$ or $\mathrm{w} / \mathrm{L}^{\alpha}$. Combining Eqs. (2) and (3) yields the following relationship between the ordinary density and the fractal density:

$$
\rho(v)=\rho_{\alpha} v^{-[1-\alpha / 3]} .
$$

Thus, the ordinary density obeys a power-law relationship with volume which has the following properties $[4][5]$ :

$$
\lim _{r \rightarrow 0} \rho=\left\{\begin{array}{l}
0, \text { if } \alpha>3, \\
\infty, \text { if } \alpha<3, \\
\rho_{\alpha}, \text { if } \alpha=3 .
\end{array}\right.
$$

In accordance with these properties, ordinary density becomes volume dependent when $\alpha \neq 3$ and it tends to either zero or infinity when the volume becomes infinitesimal. The notation of fractal density used in Eqs. (3) and (4) can be replaced by the following general model associating the fractal density and the ratio of mass and scale $(\varepsilon-$ linear size of an E-dimensional set):

$$
\rho(\varepsilon)=\rho_{\alpha} \varepsilon^{-[\mathrm{E}-\alpha]} .
$$

The power-law relation between the ordinary density and the scale is determined by two parameters: the fractal density $\rho_{\alpha}$ which is independent of scale and the exponent-singularity index $\alpha$ (fractal dimension), or $\Delta \alpha=\mathrm{E}-\alpha$; the latter also is known as the co-dimension of fractal density. The singularity index $(\Delta \alpha)$ measures the deviation of the fractal dimension from the dimension of normal density. These two parameters $\left(\rho_{\alpha}\right.$ and $\left.\Delta \alpha\right)$ can be estimated from observed data by measuring the intercept and slope of a straight line on the log-log plot of $m$ against $\varepsilon$.

\section{Fractal Integral and Fractal Differential of Fractal Density}

Integral and differential are two fundamental operations involved in modern calculus and in many other mathematical and physics subjects. The traditional integral and differentials are defined based on additive property of measure. When the measure no longer possesses additive property then the classical integral and differential may not exist. Therefore, the ordinary integral and differential operations do not applicable to the fractal density with singularity. Here I will extend the ordinary integral and differential operations to define fractal integral and differential operation as follows

$$
f_{\alpha}^{\prime}(x)=\frac{d f(x)}{d x^{\alpha}}=\lim _{v \rightarrow 0} \frac{\Delta f(x)}{\Delta x^{\alpha}},
$$

where $\Delta f(\mathrm{x})$ and $\Delta \mathrm{x}$ represent the increment of function $f(\mathrm{x})$ over an increment of $\Delta \mathrm{x}$. The convergence of the limit of form (7) can be defined as $\alpha$-fractal derivative of function $f(\mathrm{x})$. Similarly we can define the fractal integral of function $f(\mathrm{x})$ as follows 


$$
\int f(x) d x^{\alpha}=\lim _{\Delta x \rightarrow 0} \sum f\left(x_{i}\right)(\Delta x)^{\alpha},
$$

where the $f\left(\mathrm{x}_{\mathrm{i}}\right)$ is the height of function $f(\mathrm{x})$ in the small range of $\left[\mathrm{x}_{\mathrm{i}}, \mathrm{xi}_{\mathrm{i}}+\Delta \mathrm{x}\right]$. If the limit (8) converges then the limit can be named $\alpha$-fractal integral of function $f(x)$. It must remind that the fractal derivative defined in this paper is different from the fractional derivative (fractional order) available in the literature $f^{(\mathrm{v})}(\mathrm{x})$, where $\mathrm{v}$ can be an non-integer order. The fractional derivative assumes the normal integer order derivative $f^{(n)}(\mathrm{x})$ does exist. The fractal derivative is based on fractal dimension of the measure whereas the fractional derivative is based on fraction order of derivative defined on normal measure. As an example, let us take a power-law function to demonstrate the fractal derivative. Assume $f(\mathrm{x})=\mathrm{cx}^{\mathrm{b}}$, the ordinary derivative of the function $f^{\prime}(\mathrm{x})=\mathrm{cbx}^{\mathrm{b}-1}$, which does not exist at $\mathrm{x}=0$ if $0<\mathrm{b}<1$. The integral of the function is $\int \mathrm{f}(\mathrm{x}) \mathrm{dx}=\mathrm{c} /(\mathrm{b}+1) \mathrm{x}^{\mathrm{b}}+1$, does not converge if $\mathrm{b}<-1$ at $\mathrm{x}=0$. The fractal derivative at $\mathrm{x}=0$ exists and $f_{\alpha}^{\prime}(\mathrm{x})=\mathrm{c}$, if $\alpha=\mathrm{b}$. Further discussion about fractal derivative and integral will be given in separate paper.

\section{Fractal Density and Extreme Geo-processes}

In the remainder of this paper I demonstrate that fractal density $(\Delta \alpha \neq 0)$ characterizes anomalous mass accumulation or energy release caused by extreme geo-processes occurred in the Earth's crust originated from cascade earth dynamics (mantle convection and plate tectonics) and self-organized criticality (avalanches of slab breakoffs or faults). The examples to be analyzed in the rest of the paper are river flow density caused by storms or energy density released by earthquakes and intensity of earthquakes [6]. Other examples introduced in the author's previous publications include heat flow over mid-ocean ridges [2], and element concentration anomalies in surface media (stream sediments and regolith) caused by hydrothermal mineralization [7].

\subsection{Fractal density of river peak flow caused by storms}

In storm hydrology, an important phenomenon is the peak flow and recession described by the stream's discharge hydrograph, a graph showing the rate of flow (discharge) versus time past a specific point in a river. The rate of flow is typically expressed in cubic meters or cubic feet per second $\left(\mathrm{m}^{3} \mathrm{~s}^{-1}\right.$ or $\left.\mathrm{f}^{3} \mathrm{~s}^{-1}\right)$. The stream rises to peak flow after each storm event and then falls in a slow recession. Extreme flow events may occur due to accumulation of anomalous water volume in a short period time and often cause floods when the river flow exceeds the capacity of river discharge. The author's early work shows that extreme flow events depict strong local singularity that can be estimated by the accumulative average river flow [6].

$$
Q^{*}(<t)=\frac{1}{t} \sum_{i=1} Q_{i},
$$

where $Q_{\mathrm{i}}(\mathrm{i}=1,2, \ldots)$ are the river flow observed at time $\mathrm{t}_{\mathrm{i}}$ and $Q^{*}$ stands for accumulative average river flow for a time period t. At the singular location of a flow series, the quantity $Q^{*}$ follows a power-law relation with measuring unit $\mathrm{t}$ 


$$
Q^{*}(<t)=\rho_{\alpha} t^{-\Delta \alpha},
$$

where $\rho_{\alpha}$ (with unit $\left.\mathrm{m}^{3} \mathrm{~s}^{-1} / \mathrm{day}^{\alpha}\right)$ is the fractal flow rate with $\alpha$ as the fractal dimension.

This model was applied to fit the peak flow data recorded at river gauging stations in the Oak Ridges Moraine (ORM) in southern Ontario, Canada. Several gauging stations in the study area were selected for this study. These stations have records of mean daily flow $\left(\mathrm{m}^{3} / \mathrm{s}\right)$ and daily rainfall data since 1900 [8]. Figure 1A shows several gauging stations superimposed on a digital elevation model (DEM) [9]. Figure 1B shows the results obtained for the flood caused by storm after Hurricane Hazel hit the area in October 1954. The power-law model was applied to fit the observed data by least-squares method which yielded $Q^{*}=23.55 \mathrm{t}^{-0.603}, \Delta \alpha=0.603, \mathrm{R}^{2}=1.000$. Similarly, the power-law model was applied to all 17 main peak flow data from the Cataract Station on the Credit River. The values of singularity index $(\Delta \alpha)$ calculated from the 17 peak flow series range from 0.20 to 0.75 . These positive values indicate that the peak flow series each show strong singularity around the flow peak implying that the water volume released during the short period time around the peak flow is non-linearly proportional to the time duration. The singularity index may be useful for characterizing the flow in different river systems and environments. As an example, we plot the singularities calculated for the 17 events that occurred during $1900-2000$ at the Cataract Station in Figure 1C. From this plot one can see that the singularity shows general decreasing trend for the time period studied. A linear regression can be fitted to the data by least square method yielding $y=-0.003 x+6.67$, with $R^{2}=0.423$, and the student $\mathrm{t}$-value $=3.31$ (sample size $\mathrm{n}=17$ ). From this trend one can estimate the future time in about 223 years when the singularities dismiss. Of course this is based on the same decay trend and it must be kept in mind that the large error involved in the regression will lead to substantial variance of prediction. The cause of decay of singularity might be due to urban build up and man-made discharge systems in the area, evolution of the river systems and drainage networks of the area, or attributed to climate change. 

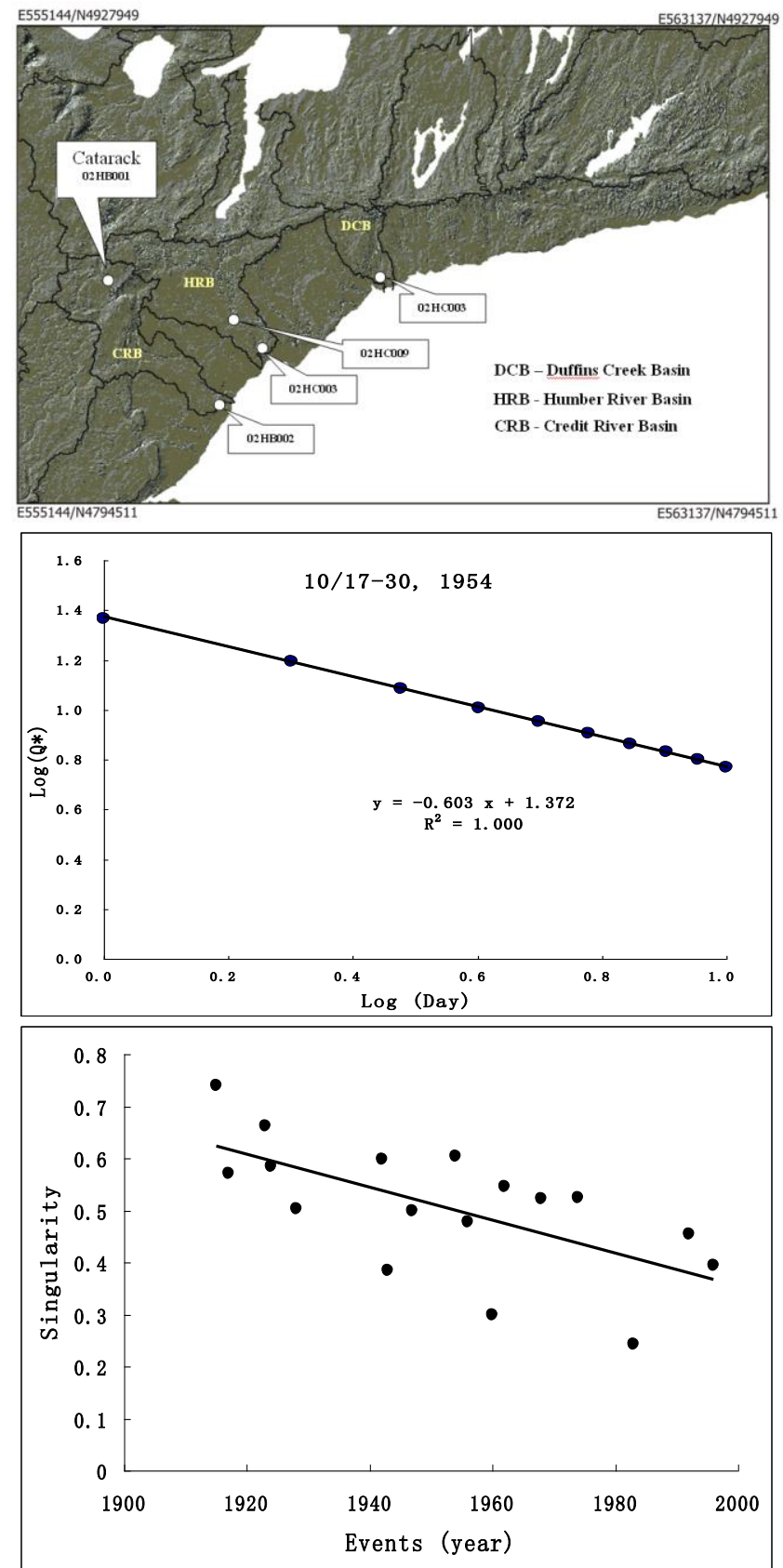

Fig. 1. (A) Shaded relief of digital elevation model of the ORM [9]. Lines represent major basins in the area. White dots represent the locations of river gauging stations chosen for the study. Labels in boxes are the IDs of gauging stations. (B) Plot shows the average flow $\left(\mathrm{Q}^{*}\right)$ as calculated for model (10). Straight line was fitted by least squares method. (C) Plot showing a general descending trend of singularity values calculated from 17 events recorded at the Cataract station. 


\subsection{Fractal energy density of intensity caused by earthquakes}

Any earthquake is due to a typical self-organized criticality (SOC) extreme process and avalanches which cause anomalous energy release within narrow spatial-temporal intervals. Patterns of the spatial, temporal and magnitude distribution of earthquakes have been the focus of many studies and the main attributes involved in models for prediction. Studies have demonstrated that the energy release of earthquakes follow power-law relations with accumulative time and scale of space. The energy released from earthquakes can be expressed as the Gutenberg-Richter equation [10]

$$
\mathrm{E}=\mathrm{E}_{0} \exp (\mathrm{bM}) \text {, }
$$

where $\mathrm{E}$ is the energy released in an earthquake, $\mathrm{E}_{0}$ is a threshold energy, $\mathrm{M}$ is the earthquake magnitude, and $b$ is a constant value. The Gutenberg-Richter equation indicates that the earthquake magnitude is a logarithmic transformation of energy release. It was proposed by Apostol [11] that the accumulative energy of earthquake and cumulative time follows a power-law relation with

$$
t / t_{0}=\left(E / E_{0}\right)^{r}
$$

where $t_{0}$ is a threshold time and $\mathrm{r}$ is a parameter accounting for the geometrical and physical properties of earthquakes. An alternative relation was proposed by Turcotte [12] to associate the energy and length scale of focal zone as

$$
E / E_{0} \approx\left(R / R_{0}\right)^{1 / r} \text {, }
$$

where $R$ and $R_{0}$ are scale lengths characterizing the focal zone. The exponent $1 / \mathrm{r}$ can be viewed as fractal dimension. This equation can be rewritten as a relation between energy and area

$$
\begin{aligned}
& E(A)=E_{0} /\left(\sqrt{A}_{0}\right)^{1 / r}(\sqrt{A})^{1 / r}=\rho_{a}(\sqrt{A})^{a}, \\
& \rho(A)=\rho_{a}(\sqrt{A})^{-(2-\alpha)}
\end{aligned}
$$

where $\rho_{\alpha}=E_{0} /\left(\sqrt{A}_{0}\right)^{\alpha}$ is the fractal energy density with $1 / \mathrm{r}=\alpha$ as the fractal dimension. It can be seen that the unit of fractal energy density $\rho_{\alpha}$ is the ratio of energy and areal scale to the power of $\alpha$ and the energy density per area, $\rho(A)$, follows power-law relationship with area.

This function was fitted to data of intensity caused by the Wenchuan earthquakes which occurred in Wenchuan, Sichuan, China, May 2008. The Mercalli intensity scale used is a seismic scale for measuring the intensity of an earthquake. It measures the overall effect of an earthquake empirically from various observed effects. The intensity of an earthquake then is not totally determined by its magnitude but incorporates its effects on people, human structures, and the natural environment. The 
National Earthquake Information Center of the United States Geological Survey has compiled and maintained an online database providing intensity maps of major earthquakes that have occurred in the world (URL: http://earthquake.usgs.gov/earthquakes/shakemap/). Figure 2 shows the distribution of intensity maps of the earthquakes including 8 aftershocks with magnitude 6 or above as occurred in Wenchuan during May-July of 2008. The Wenchuan earthquake with 8.0 magnitude that occurred at about $2 \mathrm{AM}$ on May 12 was the deadliest earthquake killing 69,197 people and leaving 18,222 missing. Strong aftershocks, some exceeding magnitude 6 , continued to hit the area even months after the main quake, causing new casualties and damage.

The data intensity and affected area for the main quake and all 8 aftershocks were fitted with power-law functions by the least-squares method. The results obtained for the main quake are estimated as, $\rho(\mathrm{A})=5377.6(\sqrt{ } \mathrm{A})^{-1.17}$, with coefficient of determination $\mathrm{R}^{2}=0.997$. Similarly, the data for 8 aftershocks were analysed with the results shown in Figure $2 \mathrm{C}$. These results indicate that the main quake with singularity $\Delta \alpha=1.17$, the first 5 aftershocks with singularity $\Delta \alpha \approx 0.83-0.88$, and the

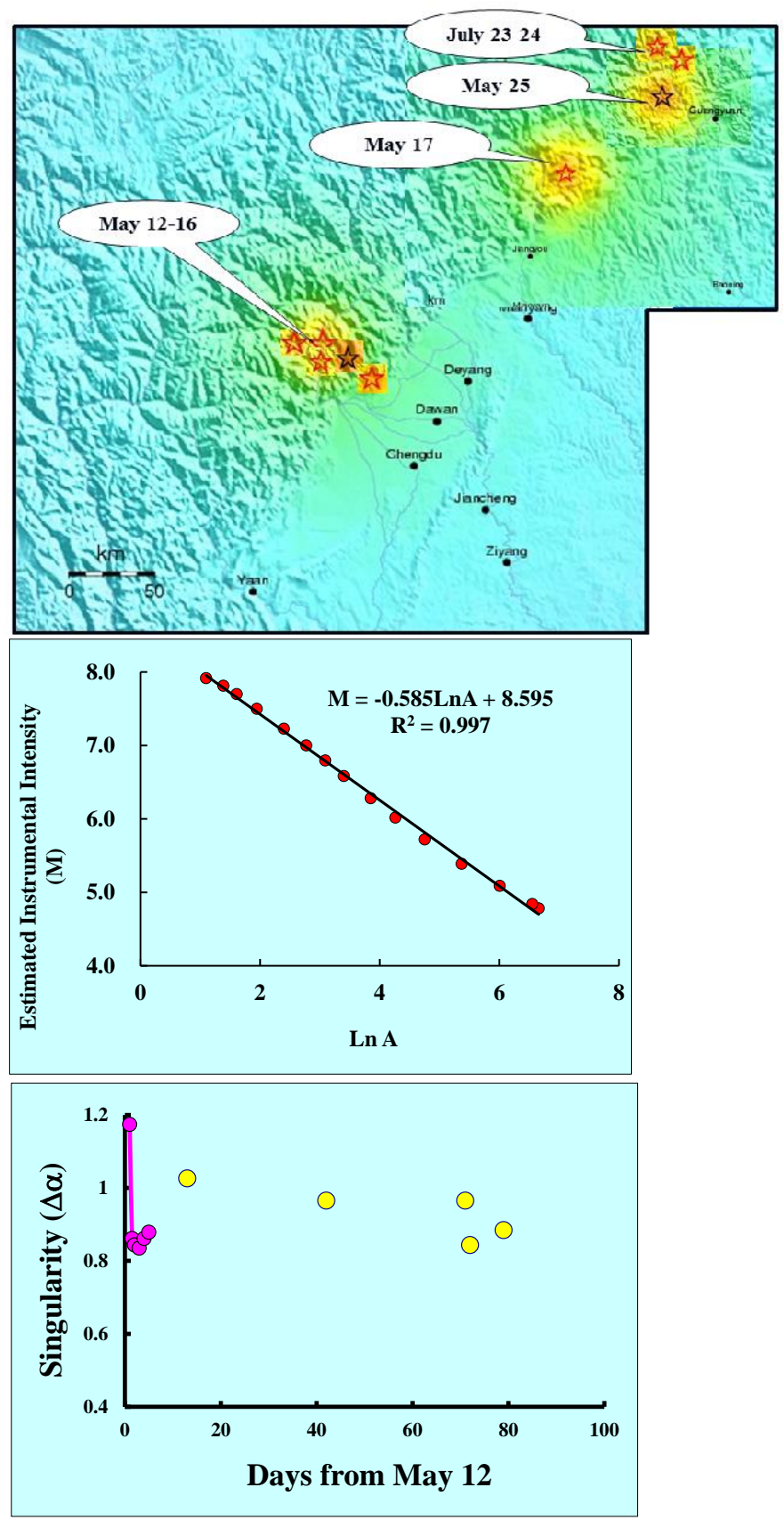

other 5

aftershocks,

which

occurred in

July, with singularity

$\Delta \alpha$ between

$0.88-1.00$.

The result

may imply

different

types of

mechanisms

were

responsible

for

immediate

aftershocks

and more

distant

aftershocks. 
Fig. 2. (A) Intensity maps showing Earthquakes with magnitude above 6 that occurred in Wenchuan, Sichuan, China, in May of 2008. An earthquake of magnitude 8 occurred on May 12, and was followed by aftershock earthquakes in May. Data were taken from the website of National Earthquake Information Centre of the United States Geological Survey (URL: http://earthquake.usgs.gov/earthquakes/shakemap/). Stars represent location of earthquakes. Color indicates the level of intensity. (B) Plot showing power-law relationship between intensity and area for the main earthquake on May 12. Red dots represent observed data and the black solid line is for model fitted by the least-squares method. (C) Plot showing the singularity indexes $(\Delta \alpha)$ estimated for the 9 earthquakes that occurred in May and June, 2008 in the same region. The results for the first 6 earthquakes are marked as purple and the other 5 as yellow for comparison. 


\section{Concluding remarks}

In this paper we have shown that the fractal density as a generalization of ordinary density can be used to characterize the singularity caused by extreme geo-processes such as those resulting in earthquakes, floods, heat flow at the mid-ocean ridges, and hydrothermal systems which occurred in the Earth's crust resulting in mineral and energy resources. The examples demonstrate that peak river flow caused by storm depicts fractal density following power-law relation with time after peak flow. Intensity caused by earthquakes depicts fractal density around epicenters with intensity decays following power-law relations for increase of area around the epicenter. Other examples shown in other papers include heat flow at mid-ocean ridges shows fractal density and the heat flow decays with distance from mid-ocean ridges following power-law relation. The element concentration in stream sediments caused by mineralization depicts fractal density and the concentration decays with distance from the center of anomalies following power-law relation. It can be generally concluded that the fractal density and local singularity analysis are applicable for most of the extreme geo-processes occurred in the Earth's crust originated from cascade earth dynamics (mantle convection) and self-organized criticality (slab breakoffs and faults as avalanches).

Acknowledgments. This research has been supported by a NSERC Discovery Research "Research and development of multifractal methods and GIS technology for mineral exploration and environmental assessments" (ERC-OGP0183993).

\section{References}

1. Mandelbrot, B.B., Multifractal Measures, Especially for the Geophysicist. Pure \& Applied Geophysis. 131: 5-42 (1989).

2. Cheng, Q.: Fractal Density and Singularity Analysis of Heat Flow in Oceanic Ridges, Scientific Report, DOI:10.1038/srep19167 (2016).

3. Cheng, Q.: Multifractal Interpolation Method for Spatial Data with Singularity. J. of the Southern Africa Institute of Mining and Metallurgy, 115, 1-6 (2015).

4. Cheng, Q.: Mapping Singularities with Stream Sediment Geochemical Data for Prediction of Undiscovered Mineral Deposits in Gejiu, Yunnan Province, China. Ore Geology Reviews. 32, 314-324 (2007).

5. Cheng, Q. and Agterberg, F.P.: Singularity Analysis of Ore-Mineral and Toxic Trace Elements in Stream Sediments. Computers \& Geosciences, 35, 234-244 (2009).

6. Cheng, Q.: Singularity Theory and Methods for Mapping Geochemical Anomalies Caused by Buried Sources and for Predicting Undiscovered Mineral Deposits in Covered Areas. J. of Geochemical Exploration, 122, 55-70 (2012).

7. Cheng, Q., Li, L. and Wang, L.: Characterization of Peak Flow Events with Local Singularity Method, Nonlinear Processes in Geophysics, 16, 503-513 (2009).

8. HYDAT CD-ROM User's Manual: Surface Water and Sediment Data, Atmospheric Environment Program, Version 96 - 1.04 User's Manual, Environment Canada, p. 95 (1996).

9. Kenny, F. M.: A Chromostereo Enhanced Digital Elevation Model of the Oak Ridges Moraine Area, Southern Ontario and Lake Ontario Bathymetry. Geological Survey of Canada. Open file 3423, scale 1:200 000 (1997). 
10. Gutenberg, B. and Richter, C.F., Frequency of earthquakes in California. Bulletin of the Seismological Society of America. 4: 185-188 (1944).

11. Apostol, B.F., A model of seismic focus and related statistical distributions of earthquakes. Physics Letters A. 357: 462-466 (2006).

12. Turcotte, D.L., Fractals and Chaos in Geology and Geophysics (2nd Edition), Cambridge Univ. Press. 398pp (1997). 